ENCYCLOPEDDIE Encyclopédie berbère

BERBERE

$31 \mid 2010$

31 | Matmora - Mezrag

\title{
Mazices, Mazaces
}

\section{Y. Modéran}

\section{OpenEdition}

Journals

Édition électronique

URL : http://journals.openedition.org/encyclopedieberbere/538

DOI : $10.4000 /$ encyclopedieberbere.538

ISSN : 2262-7197

\section{Éditeur}

Peeters Publishers

\section{Édition imprimée}

Date de publication : 30 décembre 2010

Pagination : 4799-4810

ISBN : 978-90-429-2368-3

ISSN : 1015-7344

\section{Référence électronique}

Y. Modéran, « Mazices, Mazaces », Encyclopédie berbère [En ligne], 31 | 2010, document M76, mis en ligne le 08 octobre 2020, consulté le 12 octobre 2020. URL : http://journals.openedition.org/ encyclopedieberbere/538; DOI : https://doi.org/10.4000/encyclopedieberbere.538

Ce document a été généré automatiquement le 12 octobre 2020

(C) Tous droits réservés 


\title{
Mazices, Mazaces
}

\author{
Y. Modéran
}

\section{NOTE DE L'ÉDITEUR}

Sur les usages actuels et l'étymologie berbère du terme Amazigh, voir EB IV, 1987 (notice A183,

S. Chaker).

1 De tous les ethnonymes nord-africains attestés dans l'Antiquité, Mazices est en passe, depuis une vingtaine d'années, de devenir le plus célèbre, en raison de la parenté qui semble l'unir au mot Amazigh/Imazighen*, aujourd'hui fréquemment proposé comme nom générique pour désigner l'ensemble des populations berbères. Or, écrit-on souvent au service de cette revendication, celle-ci serait d'autant plus légitime que Mazices aurait, dès l'époque gréco-romaine, déjà revêtu le même sens générique. En réalité, les sources, exceptionnellement nombreuses, ne permettent pas une affirmation aussi tranchée.

2 Aucun des auteurs anciens qui ont tenté de présenter globalement les populations autochtones d'Afrique, ou de donner une description ethno-géographique du pays, n'a en effet parlé de Mazices dans un sens aussi général. Hérodote (IV, 197) et nombre d'écrivains grecs après lui, lorsqu'ils veulent désigner les habitants de l'Afrique, emploient le terme "Libyens »* (Libues). Plus tard, les mots Gétules*, Massyles*, Numides*, et surtout Maures* furent inégalement utilisés chaque fois qu'il s'agissait d'évoquer une partie ou la totalité des populations autochtones dans une perspective très large. Mais on ne voit pas, sauf peut-être dans un ouvrage très tardif sur lequel nous reviendrons plus loin, que Mazices ait jamais tenu ce rôle dans ce genre de textes. $\mathrm{Ni}$ Salluste (qui gouverna l'Africa nova et utilisa les "livres puniques" du roi Hiempsal*), ni Strabon, ni Pomponius Mela, ni Pline (qui l'ignore manifestement, alors qu'il séjourna en Afrique et donne un tableau d'ensemble du pays), ni Ptolémée, ni Ammien Marcellin, ni Julius Honorius, ni Procope, ni Corippe* (pourtant d'origine africaine) ne l'ont employé ainsi et n'ont même semblé savoir qu'il ait pu l'être. L'auteur de la Johannide utilise, certes, comme avant lui Lucain (Pharsale, IV, 681), 
Oppien (Cynégétique, I, 270), Némésien (Cynégétique, 261), et Claudien (Eloge de Stilicon, I, 356), le mot Mazax/Mazaces, très probablement équivalent, avec une signification large. Mais tous ces auteurs ont pour point commun d'être des poètes, qui usent aussi, souvent dans les mêmes passages, d'autres termes comme Massyle ou Gétule, auxquels, au besoin, ils donnent le même sens à la fois vaste et imprécis. Loin d'employer «très fréquemment " Mazax comme on l'a écrit encore récemment, Corippe lui-même n'y recourt que sept fois (sur 4611 vers), contre 66 mentions de Maurus/Maurusius et 20 de Massylus. Et dans ces vers précis, l'emprunt à Lucain, l'un de ses modèles de prédilection, et les contraintes de la versification semblent l'avoir bien plus influencé que la volonté de tenir compte d'une réalité de son temps. Pas plus que l'existence de patronymes Mazic ou Mazuc dans l'onomastique de l'Afrique romaine, l'usage de ces termes n'est probant par lui-même. Si la possibilité que le nom ait revêtu pour les Africains un certain sens générique n'en est pas pour autant exclue, comme on va le voir, l'absence quasi totale d'occurrences de cette sorte dans tous les textes s'efforçant de donner une vue générale du peuplement de l'Afrique constitue en soi un fait qui ne peut, comme c'est trop souvent le cas, être passé sous silence.

Mazices fut, plus simplement, utilisé d'abord comme un ethnonyme spécifique, et non générique, pour désigner quelques peuplades isolées, situées en des endroits très éloignés les uns des autres. A en croire Ptolémée (IV, 1, 5), qui est le seul à le signaler, un premier groupe, situé au sud des Metagonitai, eux-mêmes proches du rivage du Détroit de Gibraltar, portait ce nom (sous la forme Masices) : on l'a situé récemment (Hamdoune 1993, p. 270-273) avec vraisemblance dans le Maroc septentrional, à l'est de Lixus, sur les premiers versants occidentaux du Rif.

Le géographe du $\mathrm{II}^{\mathrm{e}}$ siècle mentionne plus loin, dans sa description de la Maurétanie Césarienne, une seconde communauté quasi homonyme, les Mazices, qu'il place (IV, 2, 5) sous le (mont) Zelakon, lui-même au sud des Makousioi (Makhousii*), qui s'étendent vers l'est jusqu'à l'embouchure du Chinalaph: comme cette embouchure est située par le même auteur juste avant Iol-Caesarea (Cherchell) en venant de l'ouest, c'est soit dans l'Ouarsenis du Nord-Est, soit sur le versant chélifien du massif du Zaccar que ces indications nous reportent, immédiatement au nord ou au sud d'un axe Castellum Tingitanum (Chlef)-Oppidum Novum (Ain Defla)-Zucchabar (Miliana). Or c'est précisément de ces deux dernières villes que proviennent deux inscriptions mentionnant des préfets de la tribu des Mazices: l'une (AE, 1973, 654) de la fin du I I $^{\mathrm{er}}$ siècle, l'autre (sous la forme praefectus gentis Madicum, avec l'équivalence banale entre *d palatalisé et * $\mathrm{z}$ ) du IV ${ }^{\mathrm{e}}$ siècle (CIL VIII, 9613). La persistance de la tribu au Bas-Empire dans la même région est vraisemblablement confirmée aussi par les témoignages de Julius Honorius et d'Ammien Marcellin. Le premier (Cosmographie, 48), dans une liste orientée d'est en ouest, cite les Mazices après les Quinquegentiani* de Grande Kabylie et les Bures, et avant les Musunei et les Artennnites*, probables voisins d'Arsennaria (à l'ouest de Ténès), ce qui a toutes les chances de se rapporter au secteur d'Oppidum Novum et Zucchabar. Ammien Marcellin, de son côté (Histoire, XXIX, $5: 17,21,25)$ fait des Mazices un des groupes qui suivirent Firmus* dans sa révolte en 370-375. Théodose accueillit leurs envoyés à Tipasa ; il reçut ensuite comme prisonniers, alors qu'il était à Zucchabar (Sugabar), Bellen, un de leurs chefs, et Fericius, le préfet de leur tribu, tous deux mis à mort ; enfin il alla les combattre, en s'engageant dans le mont Ancorarius (Ouarsenis) et en tombant sur eux près de Tingis (Castellum Tingitanum). Toutes ces indications confirment l'existence en Césarienne, à proximité du cours moyen du Chélif, soit au nord, soit au sud, d'une seule gens des Mazices, apparemment puissante et 
pour cela surveillée de près, avec un maintien du système de la préfecture de tribu* C'est cette importance qui expliquerait aussi sa mention (sous une copie médiocre Mazaceses) dans la liste générale des grands peuples barbares donnée vers 313 par la Liste de Vérone (éd. Riese, Geographi Latini minores, p. 129), après les Quinquegentiani (Gensani sur le manuscrit) et avant les Bavares* et les Baquates*. Il est fort possible même que la garde équestre de Mazaces qui accompagnait Néron dans ses voyages selon Suétone (De vita Caesarum, Nero, XXX) ait été originaire de cette tribu.

Selon G. Camps (1960, p. 26-29), trois autres groupes de Mazices pourraient être localisés dans le sud tunisien, dans la région de Carthage, et en Numidie. En fait, les sources invoquées sont très incertaines dans les deux premiers cas. Les Maxyes* cités par Hérodote (IV, 191) à l'ouest du fleuve Triton, dans le Sud-Est tunisien donc, ont un nom qui, contrairement à ce qui est souvent dit, n'est pas exactement identique à celui des Mazices : la structure consonantique diffère, comme l'a souligné récemment R. Rebuffat (2006), et aucune considération phonétique ou morphologique ne justifie réellement le rapprochement. Le fait que ces Maxyes, équivalents peut-être aux Mazyes* d'Hécatée selon J. Desanges, sont ignorés de la quasi-totalité des autres sources anciennes incite aussi fortement à la prudence. La même attitude s'impose vis-à-vis des Maxitani, ou plutôt des Muxitani* (Desanges 1964) qui selon Justin (XVIII, 6, 1), dans un texte à forte connotation légendaire, auraient été le peuple du roi Hiarbas*, au temps du règne de Didon à Carthage. Certes, l'obstacle linguistique est ici souvent négligé, parce que, dans son commentaire d'un passage (vers 195) de la Description de la terre de Denys le Périégète (II ${ }^{\mathrm{e}}$ siècle), où est mentionnée Didon, Eustathe (éd. Müller, Geographi Graeci minores, II, p. 251), évoquant Hiarbas, le donne comme « roi des Mazices». Mais Eustathe est un savant byzantin qui écrivait au XII siècle, et nous ne savons d'où il a tiré cette information: un rapprochement arbitraire entre deux noms, ou l'utilisation d'un manuscrit défectueux ne sont nullement à exclure. Seul en fait l'emplacement en Byzacène de plusieurs toponymes Muzuc pourrait, peut-être, refléter la trace, invérifiable pour l'instant, d'une ancienne présence de Mazices dans cette province. L'hypothèse de l'existence d'un groupe de ce nom en Numidie parait, en revanche, moins fragile. G. Camps la fondait sur la mention d'évêques d'une plebs Mazacensis en 411 (Actes de la Conférence de Carthage, I, 211) et en 484 (Notitia de 484, Numidia, $\mathrm{n}^{\circ} 81$ ). Le lien de celle-ci avec une tribu est devenu plus plausible depuis la découverte en 1974 des nouvelles lettres de saint Augustin, puisque l'une d'entre elles (Lettre 22*, 1) signale la tenue au début du $\mathrm{V}^{\mathrm{e}}$ siècle d'un concile des évêques de Numidie apud Mazacos. L'identité Mazices/Mazaci est linguistiquement beaucoup mieux fondée, et on peut donc reconnaître dans cette communauté, à situer probablement dans un secteur montagneux de la Numidie du Nord, une troisième peuplade de Mazices. Comme celle-ci était apparemment bien intégrée, ce n'est sans doute pas à elle mais à la tribu attestée en Maurétanie Césarienne que doit se rapporter, au début du III siècle, à Lambèse, l'inscription (CIL VIII, 2786) d'un centurion debellator Mazicum reg(ionis) Montens(is).

Un quatrième groupe était situé nettement plus à l'est, dans les régions présahariennes ou sahariennes de la Libye actuelle, mais les sources sont ici plus difficiles à interpréter, et n'ont jamais été réellement réunies et commentées dans leur totalité. On doit souligner d'abord que les lettres 74 ou 122 de Synésios, à propos d'attaques en Cyrénaïque au début du $\mathrm{V}^{\mathrm{e}}$ siècle, ne nomment pas les Mazices : il s'agit d'une erreur due à une identification arbitraire entre Maketai* et Mazices, qui remonte à d'Avezac (Afrique ancienne, Paris, 1842, p. 135), et réapparaît pourtant encore souvent (ainsi Camps 1960, Roques 1987, Ghazi ben Maïssa 2006). Un peuple de Mazices est, en revanche, bien 
mentionné, en 359-360, par l'Expositio totius mundi et gentium (62), après « toute la région d'Afrique» et avant «la Pentapole». Comme le premier ensemble incluait la Tripolitaine, et que le second équivaut à la Cyrénaïque, ces Mazices devaient se situer apparemment dans les régions riveraines du fond de la Grande Syrte. Mais la formule de l'Expositio est ambiguë: "après toute la région d'Afrique, il y a, à ses limites méridionales, un très grand pays désert, dans une petite partie (in minima parte) duquel on dit qu'habite une gens perverse de Barbares que l'on appelle Mazices et Ethiopiens ». En fait, le texte peut se référer à n'importe quelle partie du Sahara libyen et de ses marges. On serait tenté, pourtant, de retenir la première localisation en mettant en relation l'Expositio avec un passage de l'Histoire ecclésiastique (XI, 8) de Philostorge : vers 395-399, les Mazices et les Auxourianoi, qui vivent entre la Libye et l'Afrique, désolèrent à l'est la Libye et ne détruisirent pas une partie peu importante de l'Egypte; lançant des attaques à l'ouest, ils y commirent des actes à peu près analogues. La Libye désignant à cette époque la Cyrénaïque et ses marges orientales, et l'Afrique les pays de l'ouest commençant avec la Tripolitaine, c'est en effet à nouveau vers les terres situées au sud de la Grande Syrte, qui étaient effectivement le pays des Austuriani (Auxourianoi), que ce texte nous conduit. La localisation reste cependant approximative, et la mention de raids sur l'Egypte n'est pas sans susciter de nouveaux motifs de prudence.

7 Un abondant dossier de textes concerne en effet, à partir de la fin du IV siècle et jusqu'au VI ${ }^{\mathrm{e}}$ siècle au moins, un important groupe de Mazices, qui se rendit célèbre par ses attaques fréquentes sur les monastères chrétiens établis immédiatement à l'ouest du Nil, et au moins à une occasion, entre 512 et 515, sur « la Libye » (Jean d'Antioche, fragment 216). Or dans son Histoire Lausiaque (VII, 2) écrite vers 419-420 et consacrée aux ascètes du désert de l'ouest de l'Egypte, Palladius localise assez curieusement ce peuple, en parlant, au-delà des montagnes de Nitrie, du "grand désert qui s'étend jusqu'à l'Ethiopie, les Mazices, et la Maurétanie ». La formulation rappelle celle de l' Expositio, et invite du coup à rapprocher les Mazices évoqués dans les deux textes. Pourtant ceux auxquels songe Palladius sont bien les pillards qui s'en prirent de nombreuses fois aux monastères des oasis, notamment à ceux de Scété (Wadi Natrum), et qu'évoque Jean Cassien, d'une manière dépourvue de nuances, en 426 dans sa seconde Conférence (II, 6, 2), à propos de deux moines: «ils erraient par le désert, mourant déjà de faim, lorsqu'ils furent aperçus de loin par les Mazices, une gens plus cruelle et plus inhumaine que presque toutes les autres nationes sauvages: ils ne versent pas, en effet, le sang comme d'autres par désir de butin, mais poussés uniquement par la férocité de leur esprit » (même texte dans le De virispatrum, PL, 73, col. 841-842). Dans les Verba seniorum (199, PL, 73, col. 804), le massacre « de nombreux Pères de Scété par les Mazices " est également mentionné. Plus tard, au VI $\mathrm{V}^{\mathrm{e}}$ siècle, Evagrius, en citant Nestorius dans son Histoire ecclésiastique (I, 7) à propos du séjour de ce dernier dans l'Oasis de Kharga vers 440-444, confirme la sinistre réputation de ces Mazices en Egypte: après l'avoir libéré, les Nobades, qui venaient de piller l'oasis, l'incitèrent à « fuir au plus vite la région, parce que les Mazices devaient après eux s'en emparer ». Et Jean Moschus, dans son Pré spirituel (X, 112), les montre même « ravageant et pillant toute la province ». D'autres textes (par exemple la version syriaque de l'Histoire de Jean le Petit, trad. F. Nau, Revue de l'Orient chrétien, 1914, p. 50), qui parlent d'attaques de «Libyens » ou de «Barbares » se rapportent aussi, au moins pour partie, très probablement à eux (cf. Evelyn-White 1932, p. 150-167). totalement exclu qu'il puisse être identique à celui évoqué par Philostorge et l'Expositio, 
dont les indications semblent se rapporter aux territoires syrtiques mais restent imprécises; et même si cette hypothèse est infondée, il paraît de toute façon avoir occupé un très vaste espace à l'ouest de l'Egypte et jusqu'en Cyrénaïque. Dans ces conditions, la possibilité que le terme Mazices ait possédé dans ces régions un sens large, dépassant les limites d'une gens ou même d'une confédération n'est nullement inenvisageable. Le silence à son propos des documents égyptiens du $\mathrm{III}^{\mathrm{e}}$ siècle, qui citent simplement l'attaque de "Libyens" sur l'Egypte en 258-264 (J. R. Rea, The Oxyrhynchus papyri, XLVI, Oxford, 1978, p. 46) s'expliquerait ainsi: ces derniers pourraient être les mêmes que ceux que les sources des IVe-VI siècles appellent Mazices. Faudrait-il alors remonter même plus loin, identifier aux Mazices d'autres "Libyens" voisins de l'Egypte mentionnés dans les siècles antérieurs, ou même évoquer les Meshwesh ou Mâchaouach, dont le nom apparaît pour la première fois sous Amenhotep III (1387-1348 av. J.-C.) sur des étiquettes de jarres contenant de la graisse de bœuf livrée pour la fête du Jubilé royal (K. A. Kitchen, 1990), et qui plus tard, après plusieurs attaques, finirent sous Shéshonq Ier (945-924) par occuper le trône pharaonique? L'hypothèse d'un rapport entre Meshwesh et Mazices, après d'autres, fut formulée par O. Bates (1914, p. 47), et est encore parfois reprise, mais l'équivalence n'a jamais été démontrée, et, comme l'avait déjà souligné Gsell (1927, p.354), reste extrêmement douteuse. Plus modestement, la curieuse expression de Végèce (Epitome rei militaris, III, 23) au $\mathrm{IV}^{\mathrm{e}}$ siècle qui, décrivant la tactique particulière des tribus chamelières d'Afrique au combat, conclut: " ainsi agissent les Ursiliani en Afrique, et d'autres Mazices ", s'accorderait bien, en revanche, avec un sens générique du nom, servant à désigner un vaste ensemble de peuplades du Sahara oriental. Les Ursiliani sont situés en effet par Corippus en Tripolitaine occidentale, et, en suggérant qu'eux aussi étaient des Mazices, le texte laisse supposer que l'aire de ces derniers était très étendue.

L'inventaire des sources conduit ainsi à distinguer trois groupes de Mazices précis et bien isolés les uns des autres, en Tingitane, en Césarienne centrale, et en Numidie ; et, face à eux, à l'est de la Tunisie actuelle, soit deux groupes, l'un dans les régions syrtiques et l'autre au voisinage de l'Egypte, soit un seul et vaste ensemble de Sahariens circulant de la Tripolitaine jusqu'à l'Egypte, pour lesquels Mazices aurait possédé la valeur d'un ethnonyme générique régional.

Cette répétition d'un même nom, d'un bout à l'autre du Maghreb actuel, n'est en rien banale. L'ethnographie berbère antique connait, certes, quelques cas de doublets : ainsi pour les Bavares mentionnés à la fois en Petite Kabylie et dans l'Ouarsenis, ou pour les Musuni/Musunei/Musones, attestés dans le sud-ouest de la Tunisie, au sud de Sitifis, et en Césarienne centrale. Mais une telle dispersion est réellement exceptionnelle, et, juxtaposée à la diffusion moderne du nom Amazigh, également porté par des groupes eux aussi très dispersés du Maroc à la Libye, oblige inévitablement à envisager l'hypothèse d'un sens générique initial applicable à toutes les populations autochtones de l'Afrique ancienne.

11 Cette hypothèse pourrait se construire à partir de plusieurs modèles. Déjà Gsell se demandait, très prudemment, si le mot n'avait pas été à l'origine, bien avant l'arrivée des Romains, employé effectivement par un peuple berbère conquérant, l'usage n'en ayant été ensuite conservé, au hasard des migrations et des fluctuations politiques, que par certains de ses groupes, dispersés ici et là. Si ce schéma est très hasardeux, rien n'interdit a priori de postuler, plus simplement, que Mazices ait été, depuis la fin de la protohistoire, un ethnonyme commun à tous les Berbères. Pour expliquer ensuite 
l'irrégularité ou le caractère régional de ses emplois, on pourrait supposer, en fonction d'évolutions locales diverses, la promotion dans la majorité des communautés tribales de nouveaux noms issus de sous-groupes spécifiques, et dans quelques cas la transformation par les intéressés eux-mêmes du vieux nom commun en ethnonyme particulier. Ou bien peut-être, ce qui n'a pas été proposé jusqu'ici, faudrait-il rendre responsables de cette dispersion les Grecs et les Latins : chaque fois qu'ils parlaient de Mazices à tel ou tel endroit, ils auraient, en fait, seulement retenu ce mot générique employé aussi par les autochtones, en le prenant à tort pour un nom de tribu : ainsi, par exemple, alors qu'ils savaient appeler de leur ethnonyme propre, Artennites, les habitants de la région à l'ouest de Ténès, ils se seraient contentés de qualifier leurs voisins orientaux de Mazices, nom qu'ils portaient de toute façon effectivement aussi, comme tous les autres. Dans tous les cas connus, ignorant volontairement ou non l'ethnonyme particulier, qui pourtant devait exister, ils auraient transformé le nom générique en nom tribal, parce que cet ethnonyme était difficile à prononcer, ou parce que la tribu était fractionnée en plusieurs clans, sans nom commun.

12 Toutes ces hypothèses, présentées ici par souci de n'exclure aucune possibilité, se heurtent cependant au silence des sources. Faute de textes, chercher à reconstituer la manière dont les populations de la fin de la proto-histoire se dénommaient elles-mêmes est, en fait, un exercice vain. Il faut s'en tenir aux réalités des époques historiques, et sur ce point les spéculations récentes des chercheurs modernes ont eu trop souvent le défaut de faire abstraction des conditions qui déterminent l'émergence et l'usage d'ethnonymes génériques dans une société. Le phénomène ne va pas en effet de soi, et n'est pas une donnée intemporelle de la vie des peuples. Selon sa situation politique, sociale et culturelle, une communauté ressent ou ne ressent pas le besoin de se rattacher à un ensemble plus vaste. Et le choix de l'ethnonyme commun, quand il est nécessaire, dépend aussi des circonstances, et peut varier d'une époque à l'autre : sans disparaître complètement, un nom peut se voir supplanter par un autre, tout aussi légitime aux yeux de ceux qui le portent. Or l'histoire des rapports entre Romains et autochtones en Afrique met surtout en valeur deux époques : celles où les premiers soumirent ou conquirent des royaumes, maures ou numides, puis celle où, ces royaumes détruits, ils accentuèrent par leur politique la fragmentation tribale. A aucun moment, en tout cas jusqu'à l'époque vandale, on ne voit une situation politique ou sociale qui aurait justifié le besoin de recourir, tant du côté romain que du côté africain, à un nom ethnique unique et unificateur. Si le terme Maures* prit, certes, un sens de plus en plus large à partir du II siècle, et si vers 312-313 la Liste de Vérone en fait déjà le nom commun de toutes les tribus qu'elle mentionne in Mauretania (éd. Riese, Geographi latini minores, p. 129), son usage sous cette forme resta épisodique jusqu'au $V^{e}$ siècle. Ce n'est qu'alors, lorsque les conditions politiques commencèrent à changer, que les quelques rares documents d'origine indigène que nous possédons, comme les inscriptions du roi Masuna* ou de l'imperator Masties*, montrent qu'il s'imposa aux chefs qui tentaient de réunir de larges groupements berbères indépendants derrière leur autorité : mais de Mazices, dans ces principautés de Maurétanie ou de l'Aurès, il n'est jamais question. Si ce nom avait eu un sens générique applicable à tous les Africains à la fin de la protohistoire, ce sens était apparemment oublié à la fin de l'Antiquité. Des siècles de présence romaine avaient favorisé la substitution d'un terme à un autre, et Maurus, même s'il devait son succès aux Romains d'abord, avait été assimilé et récupéré à leur profit par les autochtones (Modéran, 2004). 
13 Mais l'évolution qui s'esquissait alors fut, selon les régions, interrompue ou perturbée par la reconquête byzantine. Il fallut attendre l'invasion musulmane de la deuxième moitié du VII ${ }^{e}$ siècle pour que, dans des conditions politiques, sociales et culturelles vraiment nouvelles, se fasse ressentir la nécessité d'un ethnonyme unique pour désigner l'ensemble des populations n'étant ni grecques ni latines. L'impulsion donnée à cette évolution vint d'abord des conquérants, qui imposèrent alors le nom Berbères à la place de Mauri. Les Africains eux-mêmes participèrent cependant très vite à ce mouvement de redéfinition identitaire, dont un des vecteurs les plus remarquables fut la littérature généalogique. Celle-ci s'efforçait surtout de fixer les origines de telle ou telle qabila, mais comportait souvent une dimension plus globalisante. Or, on a depuis longtemps remarqué que surgit alors dans cette perspective un personnage qui nous ramène aux Mazices. Selon plusieurs généalogistes berbères qui vivaient au $\mathrm{X}^{\mathrm{e}}$ siècle ou au début $\mathrm{du} \mathrm{XI}^{\mathrm{e}}$ siècle (cités par Ibn Khaldûn, trad. De Slane, I, p. 169, 178, 181), notamment Sâbek ibn Sulaymân al-Matmati, Hâni ibn Mesdur al-Kûmi, et Kehlan ibn Abî Luwâ, une des deux branches du peuple berbère, les Branès*, aurait en effet eu comme ancêtre paternel un personnage nommé Mazigh, fils de Canaan, tandis que l'autre, les Butr*, descendrait du côté maternel d'une femme nommée Tamzîgh (dont le nom n'est que la forme féminine d'Amazigh). De son côté, le généalogiste arabe le plus célèbre du XI ${ }^{\mathrm{e}}$ siècle, Ibn hazm (cité et suivi par Ibn Khaldûn, III, p. 168-169 et 184), fait directement de Mazigh, toujours fils de Canaan, l'ancêtre mâle commun des deux familles berbères. Manifestement, l'apparition de cet ancêtre éponyme suppose que dès avant le $\mathrm{X}^{\mathrm{e}}$ siècle le terme Mazigh était employé par les Berbères lorsqu'ils voulaient se désigner dans leur ensemble, par delà les clivages tribaux pourtant très vifs à cette époque : et de fait, on en trouverait déjà mention (Ghazi ben Maïssa, 2006, p. 2099) de manière très brève chez un auteur du VIII ${ }^{\mathrm{e}}$ siècle, Wahb ibn Munabbih (mais on a beaucoup prêté à ce savant: cf. $E I^{2}$, t. XI, p. 38-41). Beaucoup plus tard, sans qu'il reprenne à son compte ces récits, Léon l'Africain (Description de l'Afrique, trad. Epaulard, I, p. 15), au XVI ${ }^{\mathrm{e}}$ siècle, s'accorde encore avec leur leçon essentielle, lorsqu'il signale que la langue commune des différents peuples de l'Afrique (du Nord) s'appelle aquel Amazigh [= awal amazigh].

14 Contrairement à ce qui a été souvent dit, ces légendes ne sont pas propres à l'époque arabe, et on ne peut, pour les expliquer, s'appuyer sur de mystérieuses traditions orales berbères qui auraient échappé, durant des siècles, aux Grecs et aux Latins. Elles reprennent en effet un modèle d'explication généalogique de l'origine des peuples africains aux racines bibliques très anciennes. Du côté juif, au Haut-Empire ( ?), le Targum palestinien (Néofiti 1, éd. R. Le Déaut, SC 245, p. 136-137), à propos de Genèse, X, fait ainsi figurer dans la descendance de Cham, par l'intermédiaire de Kush (et non de Canaan), au moins trois nations africaines, les Libyens, [les Zingéens], et les fils de Mauritanus, [Zemargad] et Mezag: des noms qui évoquent les Libyens, les Mauritaniens (Maures) et les Mazices (Zingéens et Zemargad se rapportent à un peuple de la Corne de l'Afrique et à une région du Sud-Est de l'Egypte). Du côté gréco-latin, on suit encore mieux le développement du modèle, à partir de la Chronique de saint Hippolyte en 235 (éd. Bauer, p. 102) et jusqu'aux Quaestiones d'Anastase le Sinaïte au VII ${ }^{\mathrm{e}}$ siècle (PG, 89, col. 558-559), en passant par le Liber Generationis I (éd. Mommsen, p. 107), la Chronique Alexandrine (ibid.), l'Ancoratus d'Epiphane de Salamine (éd. K. Holl, p. 138-139) et la Chronique Pascale (éd. Dindorf, p. 56-57). Cependant, si tous ces textes, qui ne sont également que des commentaires du Livre X de la Genèse, comportent, après avoir annoncé que la descendance de Canaan s'étend de l'Egypte à l'Océan, une mention des 
Mazices, c'est toujours aussi dans des listes plus ou moins longues d'éponymes d'autres peuples berbères, dans lesquelles figurent les Maures, les Baquates, les Gétules, les Garamantes ou les Marmarides, quand n'y surgissent pas même (Anastase le Sinaïte) les Psylles, les Musuni ou les Nasamons. L'affirmation d'un ancêtre unique porteur du nom des Mazices n'apparaît qu'avec l'époque arabe.

Un tournant se produisit donc à ce moment. On a dit plus haut que les conditions nouvelles créées par la conquête musulmane favorisèrent l'émergence d'un ethnonyme générique unique. Les Arabes imposèrent peu à peu le mot Berbères, mais du côté africain, manifestement, il semble que Mazices aurait pu prétendre alors à tenir le même rôle. Comment expliquer le succès relatif, à ce moment précis, de ce nom, dont tout ce qui précède montre qu'il n'avait jamais jusque-là possédé un sens aussi large et tenu une position aussi prééminente ? Pour le comprendre, il faut, à notre sens, traiter à nouveau le problème d'abord en historien, et revenir aux événements du VII siècle lui-même. Les premiers Berbères que rencontrèrent les Arabes en occupant l'Egypte puis ses marges, en 641-642, furent en effet d'abord les tribus de Libye orientale, c'està-dire celles que de nombreux textes des $\mathrm{IV}^{\mathrm{e}}$-VII ${ }^{\mathrm{e}}$ siècles appelaient Mazices, en donnant à ce nom un sens générique à une échelle régionale assez large. Or ces tribus, comme celles de Cyrénaïque ensuite, furent très tôt intégrées à l'armée arabe, et, associées à ses succès, acquirent un prestige et un pouvoir considérables dans tout le Maghreb. Elles s'imposèrent vite sous leurs ethnonymes spécifiques, notamment, pour certaines, celui de Lawata*, mais il est fort probable qu'elles ne perdirent jamais complètement le nom générique de Mazices, qui était aussi autrefois le leur. Et c'est donc par leur intermédiaire que ce nom aurait connu alors une seconde jeunesse et une extension nouvelle, et par là gagné ensuite dans les généalogies tribales un rang particulier (même si elles-mêmes cherchèrent plus tard à s'en éloigner en s'inventant des origines arabes).

Peut-être même eut-on quelques échos en Occident de cette évolution. Ici doit être cité en effet, pour finir, le seul texte latin qui valorise vraiment les Mazices : un passage d'une description du monde très tardive, au minimum postérieure au $\mathrm{V}^{\mathrm{e}}$ siècle, la Cosmographia du Pseudo-Aethicus, une sorte de manuel scolaire qui reprend en le modifiant le texte de Julius Honorius (éd. Riese, Geographi Latini minores, p. 88). Tout en adoptant comme son modèle une division du monde en quatre "océans", lorsqu'il aborde «l'océan méridional», l'auteur, dans le sommaire annonçant le nombre de peuples, emploie, au lieu de gentes XXIII, cette formule: gentes Mazices multas ( $« \mathrm{de}$ nombreuses tribus Mazices»). Cette expression n'a aucun équivalent chez Julius Honorius, ni chez Orose, l'autre source identifiable de la Cosmographia, et constitue un ajout très original, qui parait bien impliquer l'usage d'un sens générique de Mazices étendu à l'échelle de l'ensemble de l'Afrique. Malheureusement, en dehors de la datation au VIII siècle du plus ancien manuscrit de l'œuvre, nous ignorons tout de l'auteur de la Cosmographia, et du lieu où il écrivit. Quelques indices sont cependant troublants. P. Gautier-Dalché (Nicolet et Gautier-Dalché, 1986, p. 193, n. 37) a déjà noté que plusieurs détails de sa description de Rome «évoquent les itinéraires romains du $\mathrm{VII}^{\mathrm{e}}$ siècle». On ajoutera l'absence, dans le cours du développement, d'une liste nominale de ces gentes Mazices multae (alors qu'on trouve respectivement 51, 34 et 30 noms de gentes pour les trois autres parties du monde). Le Pseudo-Aethicus se contente en effet d'écrire: "l'Océan méridional a d'innombrables peuples et il n'est pas possible de les dénombrer, ni d'avoir une opinion sur elles ou de s'exprimer à leur propos, à cause des déserts qui s'interposent ». Or il faut ajouter à cette remarque un autre passage original au début 
de l'œuvre, où l'auteur refuse de considérer l'Afrique comme la troisième partie du monde, sur le même plan que l'Europe et l'Asie, en raison de sa "très mauvaise situation » (situ pessimo). Tout ceci, dans un texte par ailleurs si fidèle à Julius Honorius, paraît bien refléter la vision d'une époque où on ne s'intéressait plus à l'Afrique, parce que l'évolution de ses populations les rendait définitivement étrangères au monde occidental. Une datation à la fin du VII siècle n'aurait rien d'étonnant.

La longueur de cette notice a été justifiée plus haut par l'enjeu que représente l'histoire des Mazices depuis quelques années, avec le développement du mouvement revendiquant la promotion du nom Amazigh. Or, en définitive, à travers surtout un type de sources (les généalogies, et non les descriptions géographiques ou ethnographiques), ce nom ne nous parait avoir acquis l'importance que ses promoteurs actuels lui prêtent qu'à partir du VII ${ }^{e}$ siècle, lorsque la conquête arabe créa des circonstances politiques et culturelles qui favorisèrent l'émergence d'un ethnonyme générique. Auparavant, il n'est mentionné avec certitude qu'à partir du premier siècle, et servit longtemps à désigner diverses peuplades établies du Maroc aux frontières de l'Egypte, sans lien entre elles. Cette dispersion d'un même nom reste en elle-même énigmatique. Il est fort possible qu'elle reflète un usage ancien, plus général, sur lequel malheureusement ne peuvent être formulées que de pures hypothèses. Le succès (relatif) du nom des Mazices à partir du $\mathrm{VIII}^{\mathrm{e}}$ siècle, quant à lui, semble bien avoir reposé surtout sur l'expansion vers l'ouest, à la faveur de l'alliance avec les Arabes et d'une islamisation précoce, des tribus libyennes pour lesquels ce nom de Mazices revêtait déjà un sens générique régional au Bas-Empire.

\section{BIBLIOGRAPHIE}

BASSET R., Le dialecte de Syouah, Paris, 1890.

BATES O., The Eastern Libyans. An Essay, Londres, 1914.

CAMPS G., « Massinissa ou les débuts de l'histoire », Libyca, VIII, 1, 1960, p. 1-320.

CoLIN F., Les peuples libyens de la Cyrénaïque à l'Egypte, Bruxelles, 2000.

DESANGES J., Catalogue des tribus africaines de l'Antriquité classique à l'ouest du Nil, Dakar, 1962.

DESANGES, J., « Rex Muxitanorum Hiarbas (Justin, XVIII, 6, 1) », Philologus, CXI, 1967, p. 304-308.

EVELYN WHITE H. G., The Monasteries of the Wâdi'n Natrûn, tome 2, The History of the Monasteries, New York, 1932.

GHAZI BEN MAÏSSA H, « Et si on appelait ces fameux Berbères par leur vrai nom ? », L'Africa romana XVI, Atti del XVI convegno di studio, Rabat, 15-19 dicembre 2004, t. 3, Rome, 2006, p. 2089-2108.

GSELL, S., Histoire ancienne de l'Afrique du Nord, tome V, Paris, 1927.

HAMDOUnE C., « Ptolémée et la localisation des tribus de Tingitane », MEFRA, 105, 1, 1993, p. 241-289. 
KITCHEN K. A., " The Arrival of the Libyans in Late New Kingdom Egypt », dans A. Leahy, Libya and Egypt, 1300-750, Londres, 1990, p. 15-27.

LEVEAU Ph., «L'aile II des Thraces, la tribu des Mazices, et les praefecti gentis en Afrique du Nord », Antiquités africaines, 7, 1973, p. 153-192.

MODERAN Y., Les Maures et l'Afrique romaine IV ${ }^{e}$-VII ${ }^{e}$ siècle, Rome, 2003.

MODERAN Y., « Les Maures de l'Afrique romaine dans l'Antiquité tardive », Revue des études latines, t. 82,2004 , p. 249-269.

NICOLET C., GAUTIER DALCHE P., « Les quatre sages de Jules César et la mesure du monde selon Julius Honorius : réalité antique et tradition médiévale », Journal des Savants, 1986, p. 157-218.

REBUFFAT R., « Notes d'onomastique ethnique. Les Maces », L'Africa romana XVI, Atti del XVI convegno di studio, Rabat, 15-19 dicembre 2004, t. 1, Rome, 2006, p. 403-444.

ROQUES D., Synésios de Cyrène et la Cyrénaïque du Bas-Empire, Paris, 1987.

WAGNER, Les oasis d'Egypte à l'époque grecque, romaine et byzantine d'après les documents grecs, Bibliothèque d'études de l'IFAO, Le Caire, 1987.

INDEX

Mots-clés : Antiquité, Ethnonymie, Peuplement, Population, Tribu 